\title{
Positive impact of fibronectin in stimulation human adipose-derived mesenchymal stem cells attachment seeded into polytetrafluoroethylene patch for future surgical closure of atrial and ventricular septal defect
}

\author{
I Gde Rurus Suryawan ${ }^{1 *}$, Anudya Kartika Ratri ${ }^{1}$, Andrianto Andrianto ${ }^{1}$, Meity \\ Ardiana ${ }^{1}$, Ricardo Adrian Nugraha ${ }^{1}$ \\ Department of Cardiology and Vascular Medicine, Faculty of Medicine \\ Universitas Airlangga - Dr. Soetomo General Hospital, Surabaya - \\ INDONESIA \\ * Corresponding author: I Gde Rurus Suryawan, M.D., Ph.D. \\ Corresponding address: Mayjend Prof. Dr. Moestopo 6-8, Surabaya - \\ INDONESIA \\ Corresponding email: igde.rurus.s@fk.unair.ac.id
}

Background. Polytetrafluoroethylene (PTFE) patch is commonly used during surgical closure for atrial septal defect (ASD) and/or ventricular septal defect (VSD). It has several limitations such as inability to grow, repair, and remodel. Aneurysm formation, thrombosis, and the inability of patches to grow or remodel are usual, especially in children and young adults. To tackle these limitations, we try to use fibronectin and human adipose-derived mesenchymal stem cells (hAMSCs) in PTFE patch.

Objective. To understanding positive impact of fibronectin to enhance hAMSCs cell-to-cell adherence and cell-to-patch surface attachment into PTFE patch for future ASD or VSD closure.

Methods. Cultured of hAMSCs cells were fixated with $15 \mathrm{~mL}$ methanol and CD90+, CD105+, CD45- antibodies were labeled FITC, rinsed with PBS and analyzed under fluorescence microscope for 15 minutes. Fibronectin solution $0.1 \%$ were used to soak patch scaffolds for approximately 2 hours duration, and then dried for 20 minutes for treatment group. As for control group, Fibronectin solution was not added on the culture. The samples were examined with scanning electron microscope (SEM).

Results. SEM examination showed incomplete attachment of the cells even after 10 days on control group at $1.14 \pm 1.13$ (Figure 2). In contrast, treatment group showed more cells attached to the patch surface at $31.25 \pm 13.28$ ( $p$ 0.000) (Figure 3). Observation at 5 days was $17.67 \pm 20.21$, at 7 days was $12.11 \pm 10.94$, at 10 days was $18.83 \pm 23.25$. No significant statistical difference of mean cell per view among each treatment group ( $p$ 0.802).

Conclusion. Fibronectin has a positive impact on hAMSCs attachment seeded onto PTFE patch. These properties, in combination with their developmental plasticity, have generated tremendous interest because of the potential use of hAMSCs in regenerative medicine to replace damaged tissues.

Keywords: ASD, fibronectin, hAMSCs, PTFE patch, VSD 


\section{INTRODUCTION}

Acyanotic congenital heart disease (CHD) such as Atrial Septal Defect (ASD) and Ventricular Septal Defect (VSD) are the most common CHD (50\% of all CHD). They are often underdiagnosed because of lack of symptoms and only $20 \%$ of patients will require surgical intervention (1). Usually, a patch of fabric or pericardium is sewn over the ASD or VSD to close it completely. Unfortunately, materials used for patch repair or reconstruction have limitations such as their inability to grow, repair, and remodel. Aneurysm formation, thrombosis, and the inability of patches to grow or remodel are important sources of morbidity and mortality after repair or reconstruction of cardiovascular structures, especially in children and young adults (2). Tissue engineering has become an evolving in vitro innovative approach for intended surgical replacement of congenital defects. By transplanting multi potential human adipose derived mesenchymal stem cells (hAMSCs) onto widely used PTFE patch, authors offer a new solution for its prosthetic limitations. Fibronectin as an extracellular matrix protein was added to enhance cell-to-cell adherence, and cell-to-patch surface attachment.

\section{Objective}

To understanding positive impact of fibronectin to enhance hAMSCs cell-to-cell adherence and cell-to-patch surface attachment into PTFE patch for future ASD or VSD closure. 


\section{MATERIALS AND METHODS}

\section{Scaffold materials}

Cardiovascular synthetic patch made of polytetrafluoroethylene/PTFE (GoreTex®) was used. It is a durable, easy to handle, sterile synthetic patch designed for optimal tissue ingrowth while minimizing aneurysmal dilation in a wide variety of cardiovascular applications including cardiac, great vessel, and peripheral vascular reconstructions and surgeries. The patch was minced into $0.8 \times 0.8 \mathrm{~cm}^{2}$ pieces.

\section{Mesenchymal stem cells culture and harvesting}

Mesenchymal stem cells obtained by thawing of cryopreserved human adipose derived mesenchymal stem cells. They were thawed at $37^{\circ} \mathrm{C}$ and transferred in $5 \mathrm{~mL}$ of standard cell media and gently homogenized. $500 \mu \mathrm{L}$ warm phosphate buffered saline (PBS) added into medium well for 2 minutes. Resuspension with PBS plus $500 \mu \mathrm{L}$ Trypsin / EDTA solution (0,5\%) and incubated for 5-10 minutes $37^{\circ} \mathrm{C}$ and $5 \%$ of $\mathrm{CO}_{2}$ until they reached $80 \%$ of confluence. The cell suspension was transferred for sterile centrifuge tube and centrifuged at $300 \mathrm{~g}$ for 5 minutes. After centrifugation, $200 \mu \mathrm{L}$ warm PBS solution was added to each centrifuge tube. After another centrifugation, the supernatant was aspirated, and the cells were resuspended in approximately $500 \mu \mathrm{L}$ of stromal medium. $1 \mathrm{~mL}$ aliquot cell dilution in trypsin blue were submitted to counting cells using hematocytometer. The cells then placed onto suitable culture plates. For each sample, the culture was done at passage 5. Using light microscope, the cell culture showed spindle-like shaped morphology consistent with mesenchymal stem cell. 


\section{Identification of hAMSCs phenotype}

All samples were analyzed for the expression of surface markers, using monoclonal antibodies against cluster of differentiation (CD) antigens, conjugated with fluorochromes, and analyzed after thawing by indirect immunofluorescence. Cultured cells were fixated with $15 \mathrm{~mL}$ methanol and CD90+, CD105+, CD45- antibodies were labeled FITC, rinsed with PBS and analyzed under fluorescence microscope for 15 minutes.

\section{Fibronectin addition onto PTFE patch surface}

Fibronectin solution $0.1 \%$ were used to soak patch scaffolds for approximately 2 hours duration, and then dried for 20 minutes for treatment group. As for control group, Fibronectin solution was not added on the culture.

\section{hAMSCs direct seeding into PTFE patch}

1-5 $\mu$ l of solution is aspirated using micropipette, approximately $2 \mu \mathrm{L}( \pm 10.000$ cells) were seeded onto PTFE patch surface in M-96 medium wells. the cells were cultured in Dulbecco's Modified Eagle Medium (DMEM [Gibco Life Technologies]) supplemented with 10\% fetal calf serum (FCS [Sigma-Aldrich, Germany]) and $1 \%$ penicilin-streptomycin-glutamine solution (Gibco Life Technologies). The medium was changed every 2 days. The cell-seeded scaffolds were placed in 96 medium wells under dynamic cell culture conditions and divided into for 5-, 7- or 10-days observation groups. After the required observing days, the samples were harvested and prepared for scanning electron microscope (SEM) examination. The analysis of patches includes 
qualitative and quantitative examination by counting mean number of cells per field of view.

\section{Structure Examination with SEM}

The samples were fixed with $2 \%$ Glutaraldehyde, followed by gradual dehydration using alcohol. Afterward, the specimens were critical point dried in CO2 using a Hitachi HCP-2 critical point dryer (Hitachi, Tokyo, Japan) and was subsequently gold sputtered using vacuum evaporator. The samples were examined with Jeol scanning electron microscope (Hitachi, Tokyo, Japan) and the images were captured by Nikon camera.

\section{Statistical Analysis}

Data results were expressed as mean $\pm S D$. Data and graphs were produced with MS Excel 2010 (Microsoft Corp, USA). Comparisons between the Fibronectin added hAMSCs seeded patch and control group were performed with Mann Whitney $U$ test and one way ANOVA test using SPSS Statistics 25.0 (IBM Corp, USA). Statistical significance was set at $p$ less than 0.05 . 


\section{RESULTS}

International Society for Cellular Therapy clearly stated a criteria for minimum expression of $h$-AMSCs immunophenotyping, included CD73+, CD90+, CD44+ dan CD105+, and CD45- negative expression based on phase 2 Immunocytochemistry (ICC). In our samples, data show a positive expression for CD105+ and negative expression for CD45- based on Immunocytochemistry (Figure 1).

Scanning electron microscopy examination showed incomplete attachment of the cells even after 10 days on control group at $1.14 \pm 1.13$ (Figure 2 ). In contrast, treatment group showed more cells attached to the patch surface at $31.25 \pm 13.28$ ( $p$ 0.000) (Figure 3). Observation at 5 days was $17.67 \pm 20.21$, at 7 days was $12.11 \pm 10.94$, at 10 days was $18.83 \pm 23.25$. No significant statistical difference of mean cell per view among each treatment group ( $p$ $0.802)$ 


\section{DISCUSSION}

Stem cell therapy in tissue engineering has been a promising strategy to reconstruct and as a unique replacement of congenital defects. The important components are the cells used, scaffold material and supportive signaling molecules. hAMSCs has been recommended by International Fat Applied Technology Society for having good differentiation capacity, easy accessibility in terms of isolation procedure, and the proliferation ability does not change throughout patient's life (3). Therefore, hAMSCs were used in this study for their superiority over bone marrow MSCs.

Many factors affect the efficacy of solubilized protein such as Fibronectin on surfaces the distance between the protein and substratum (4-5); the immediate environment surrounding the functional domain (6); the configuration of the protein and its secondary structure (7). Those factors may affect the accessibility of the active part of the protein to the cell, the activity of the peptide and topography of the surface.

PTFE patch has low seeding efficiency and difficulty to withhold cells (8), this might be due to hydrophobic and lightweight character of the patch itself, not allowing to sink into the culture media. Adsorption or immobilisation of specific ECM proteins can therefore be used to enhance cell attachment. In order to mimic the in vivo environment, purified components of the ECM are frequently used in the laboratory to coat cell culture plastic or glass to enhance cell adhesion (9). Cell culture studies using human coronary artery smooth muscle cells onto polyurethane scaffold demonstrated that Fibronectin-conjugated 
scaffolds had improved cell attachment and infiltration depth compared with scaffolds without Fibronectin conjugation and with those scaffolds on which Fibronectin was merely adsorbed at 4 days cell culture (10). This study shows that undifferentiated hAMSCs seeded onto those patch scaffolds can attach more with Fibronectin addition. On the other hand, the control group showed the original structure of the patch rather than homogenous and confluent surface.

Smaller size of scaffolds and many greater numbers of cells used to seed may improve the attachment and retainability of cells onto the patch surface. However, the molecular interactions between hAMSCs and Fibronectin protein remain unknown.

The longer duration of cell culture did not show any statistically significant difference. This may imply that after Fibronectin addition, 5 days of cell culture has shown good cell distribution, well cell attachment onto the patch surface as expected. The findings of this early study may add an important point to create biomimetic patch in vitro. However, the potency of hAMSCs seeded on patches to induce differentiation into grown cardiac endothelial cells still needs further research. Its' physical and chemical durability still needs further evaluation in terms of extracellular matrix formation and endothelial coverage before expected clinical application in the future. 


\section{CONCLUSION}

Fibronectin has a positive impact on hAMSCs attachment seeded onto PTFE patch. These properties, in combination with their developmental plasticity, have generated tremendous interest because of the potential use of hAMSCs in regenerative medicine to replace damaged tissues. Longer duration of cell culture did not provide significant difference on cell-to-patch surface attachment.

\section{AUTHOR CONTRIBUTIONS}

A.K.R. conceived and planned the experiments, carried out the experiments, planned and carried out the simulations, contributed to sample preparation. I.G.R.S. contributed to the project administration and funding. A.A. contributed to the interpretation of the results. R.A.N. initially writing the manuscript. All authors provided critical feedback and helped shape the research, analysis, and manuscript.

\section{ACKNOWLEDGEMENT}

We would like to express special gratitude to all staff and residents from Department of Cardiology and Vascular Medicine Universitas Airlangga as well as all staff from Institute of Tropical Diseases Universitas Airlangga who allowed us to do this research. We would like to Budi Santoso as Dean of Faculty of Medicine Universitas Airlangga and Djoni Wahyuhadi as Director of Dr. Soetomo General Hospital for their support in this research. 


\section{FINANCIAL SUPPORT AND SPONSORSHIP}

Nil.

\section{CONFLICT OF INTEREST}

The authors declare that they have no competing interests.

\section{AVAILABILITY OF DATA AND MATERIAL}

The data that support the findings of this study are available from the corresponding author upon reasonable request. 


\section{REFERENCES}

1. Hoffman JIE, Kaplan S, Liberthson RR. (2004) Prevalence of congenital heart disease. Am Heart J. 2004;147:425-39.

2. Yang C MD et al. (2006) In Vitro Fabrication of a Tissue Engineered Human Cardiovascular Patch for Future Use in Cardiovascular Surgery. Ann Thorac Surg 2006;81:57-64

3. Zuk PA, Zhu M, Mizuno H, Huang J, Futrell JW, Katz AJ, Benhaim P, Lorenz HP, Hedrick MH (2017), Multilineage cells from human adipose tissue: implications for cell-based therapies. Tissue Eng 2001;7:211228.

4. Houseman BT, Mrksich M (2001) The microenvironment of immobilized Arg-Gly-Asp peptides is an important determinant of cell adhesion. Biomaterials 22:943-955

5. Tong YW, Shoichet MS (2001) Enhancing the neuronal interaction on fluoropolymer surfaces with mixed peptides or spacer group linkers. Biomaterials 22:1029-1034

6. Dori Y, Bianco-Peled H, Satija SK, Fields GB, McCarthy JB, Tirrell M (2000) Ligand accessibility as means to control cell response to bioactive bilayer membranes. J Biomed Mater Res 50:75-81

7. Ochsenhirt SE, Kokkoli E, McCarthy JB, Tirrell M (2006) Effect of RGD secondary structure and the synergy site PHSRN on cell adhesion, spreading and specific integrin engagement. Biomaterials 27:38633874

8. Rosenman JE, Kempczinski RF, Pearce WH, Silberstein EB (1985) Kinetics of endothelial cell seeding. J Vasc Surg 1985;778-84 
9. Kleinman HK, Luckenbill-Edds L, Cannon FW, Sephel GC (1987) Use of extracellular matrix components for cell culture. Anal Biochem 166:1-13

10. Dubey G, Mequanint K (2011) Conjugation of fibronectin onto threedimensional porous scaffolds for vascular tissue engineering applications. Acta Biomaterialia 7 (2011) 1114-1125 


\section{FIGURE LEGENDS}
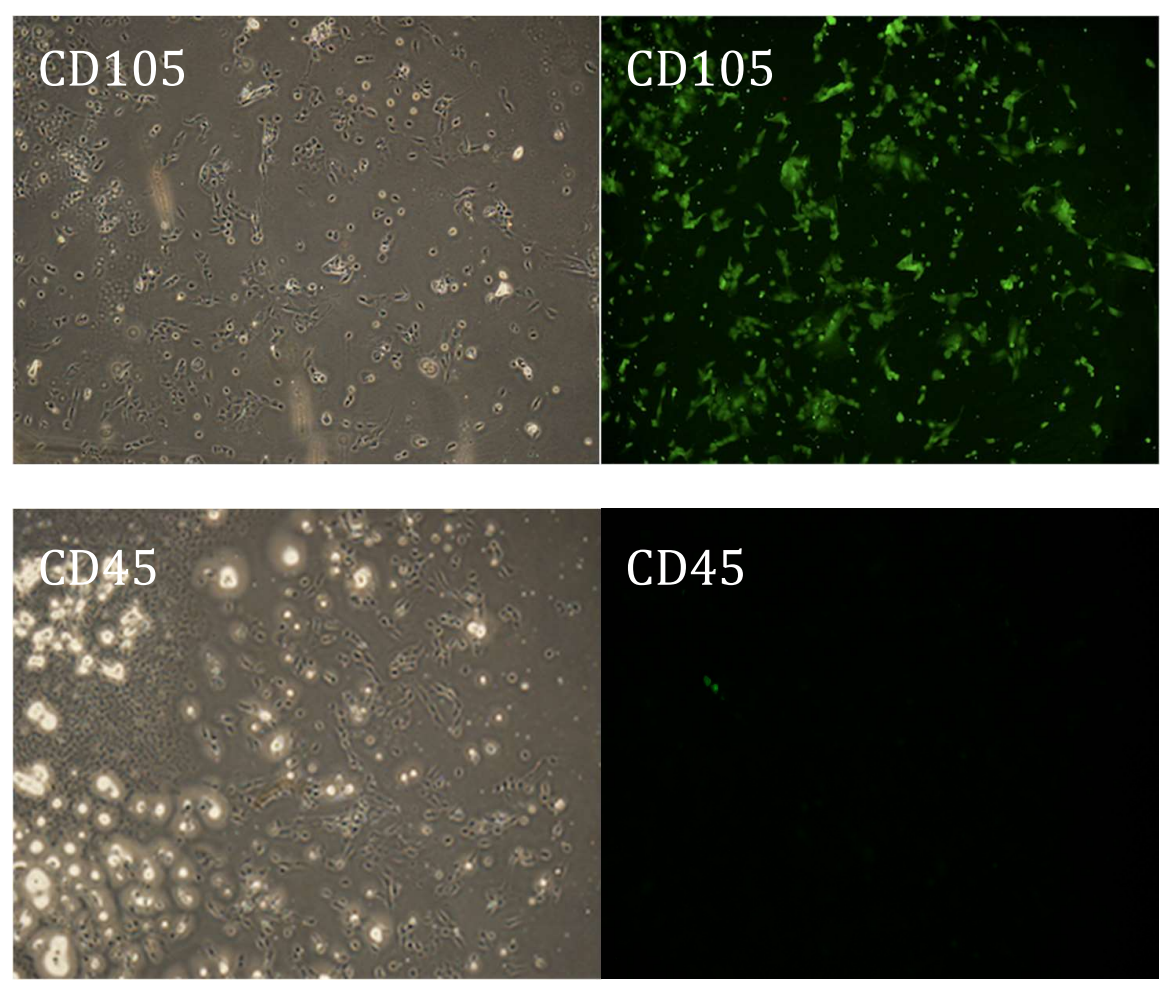

Figure 1. Cell cultures characteristic based on Immunocytochemistry (ICC) CD90+ and CD105+ (positive expression showed green luminescence) whereas CD45- (negative expression doesn't show) 


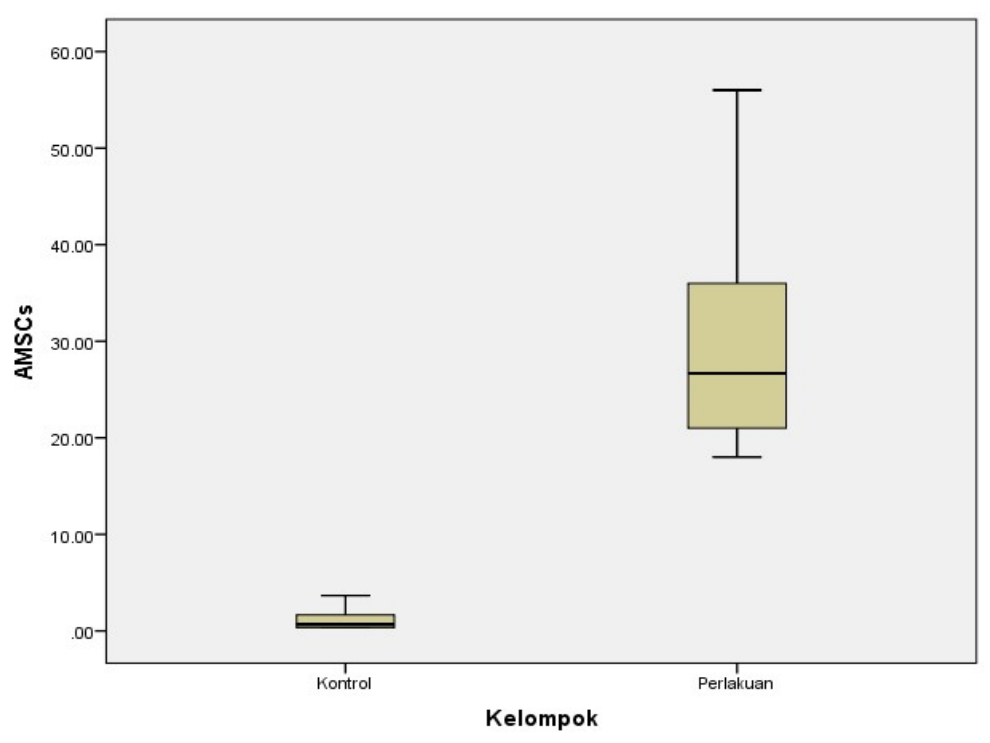

Figure 2. hAMSCs attachment onto PTFE patch surface: control vs treatment group (mean number of cell per field of view) 

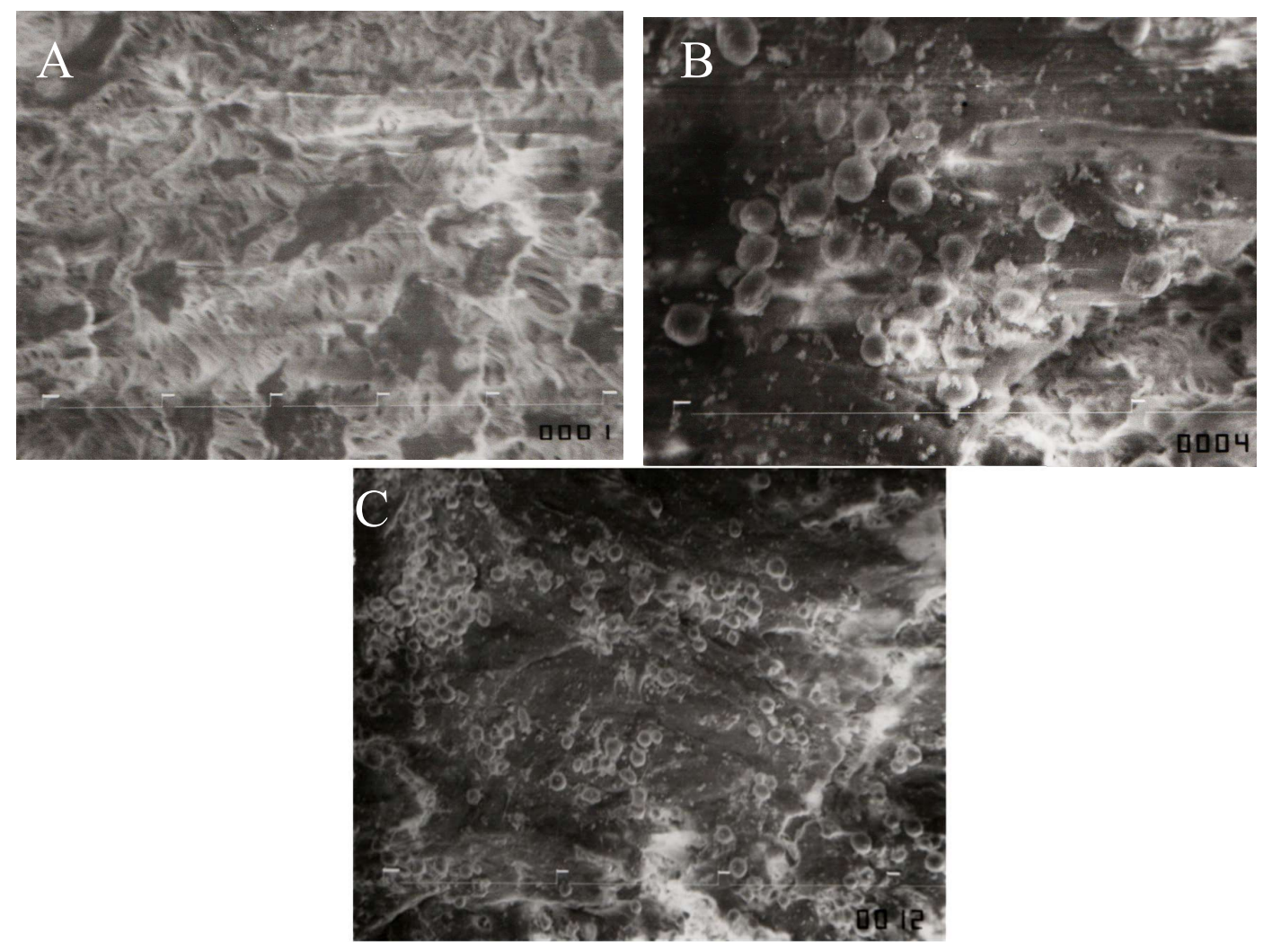

Figure 3. Scanning electron micrographs: control group without Fibronectin addition (A); treatment group after 5 days $(B)$ (magnification 1000x); treatment group after 5 days (C) (magnification 350x) 